\title{
Hinweise auf glykämische Schwellenwerte bei Typ-2-Diabetikern
}

Fragestellung: Gibt es bei der ADVANCE-Studie $\mathrm{HbA}_{1 \mathrm{c}}$-Schwellen, über denen das Risiko für mikro- und makrosvaskuläre Komplikationen ansteigt oder abfällt?

Hintergrund: Derzeit gibt es widersprüchliche Empfehlungen für $\mathrm{HbA}_{1 c^{-}}$Zielwerte bei Patienten mit Typ2-Diabetes. Die ACCORD-Studie wurde vor ein paar Jahren abgebrochen, nachdem die drastische, kurzfristige Senkung des $\mathrm{HbA}_{1 \mathrm{c}}$ bei kardiovaskulären Risikopersonen und langjährigen Typ-2-Diabetikern mit einer erhöhten Mortalität einherging. In der vorliegenden Studie wurde der Zusammenhang zwischen $\mathrm{HbA}_{1 \mathrm{c}}$ und dem Risiko vaskulärer Komplikationen und Tod bei der ADVANCE-Studie untersucht.

Patienten und Methodik: Im Rahmen der ADVANCE (Action in Diabetes and Vascular disease: Preterax and Diamicron Modified Release Controlled Evaluation)Studie wurden 11140 Patienten randomisiert einer intensiven oder standardisierten antidiabetischen Therapie zugeteilt. Der mittlere $\mathrm{HbA}_{1 \mathrm{c}}$-Wert wurde wiederholt während der Beobachtungszeit und vor dem ersten Ereignis bestimmt. Adjustierte Risiken für jede $\mathrm{HbA}_{1 c^{-}}$ Dezile wurde mit COX-Regressionsmodellen erstellt. Mögliche Unterschiede der Assoziation von $\mathrm{HbA}_{1 \mathrm{c}}$ und Risiko bei unterschiedlich hohem $\mathrm{HbA}_{1 \mathrm{c}}$ wurde mithilfe „Linear spine“-Modellen analysiert.

Ergebnisse: Die Assoziation von mittlerem $\mathrm{HbA}_{1 \mathrm{c}}$ im Verlauf und dem Risiko für makro- und mikrovaskuläre Ereignisse und Tod war nicht linear. Innerhalb des untersuchten $\mathrm{HbA}_{1 \mathrm{c}}$-Bereiches (5,5-10,5\%) gab es Hinwei- se auf „Schwellenwerte“. Es zeigte sich, dass sich das Risiko bei $\mathrm{HbA}_{1 \mathrm{c}}$-Veränderungen unterhalb von $7 \%$ für makrovaskuläre Ereignisse und Tod, und $\mathrm{HbA}_{1 \mathrm{c}}$ unter 6,5\% für mikrovaskuläre Ereignisse nicht signifikant veränderte $(p>0,8)$. Oberhalb dieser Schwellenwerte stieg das Risiko signifikant an: Mit jedem Anstieg des $\mathrm{HbA}_{1 \mathrm{c}}$ um $1 \%$ stiegen das Risiko für ein makrovaskuläres Ereignis um $38 \%$, das Risiko für ein mikrovaskuläres Ereignis um $40 \%$ und das Risiko für Tod um 38\% an (alle $\mathrm{p}<0,0001$ ).

Schlussfolgerung: Bei Patienten mit Typ-2-Diabetes sind $\mathrm{HbA}_{1 \mathrm{c}}$-Werte bis $7 \%$ mit weniger makrovaskulären Ereignissen und $\mathrm{HbA}_{1 \mathrm{c}}$-Werte bis $6,5 \%$ mit weniger mikrovaskulären Ereignisse assoziiert. Unterhalb dieser Schwellen gab es weder Hinweise für ein weiter erniedrigtes Risiko noch für ein erhöhtes Risiko.
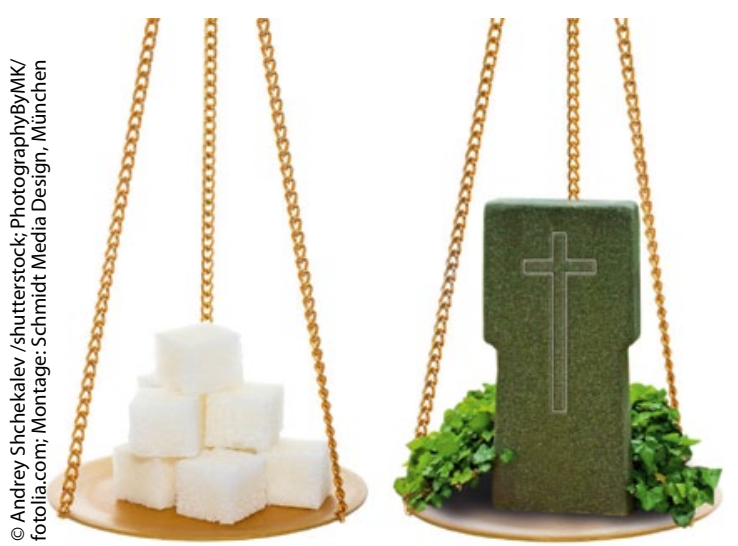

Zoungas S, Chalmers J, Ninomiya T et al. Association of $\mathrm{HbA} 1 \mathrm{c}$ levels with vascular complications and death in patients with type 2 diabetes: evidence of glycaemic thresholds. Diabetologia 2012;55(3):636-43.


PD Dr. med. Nanette C. Schloot
PD Dr. med. Nanette C. Schloot

Deutsches Diabetes-Zentrum,

Institut für Kinische Diabetologie Düsseldorf Klinik für Stoffwechselkrankheiten, Uniklinikum Düsseldorf Medical BU Diabetes, Lilly Deutschland GmbH Werner-Reimers-Str 2-4, D-61352 Bad Homburg schloot@ddz.uni-duesseldorf.de zusätzlichem Nutzen (oder Schaden) kam. Wenn wir die Patienten also mit $\mathrm{HbA}_{1 \mathrm{c}}$-Zielwerten von $6,5 \%-7,0 \%$ einstellen, und dies nicht mit zu „rabiaten“ Methoden wie in der ACCORD-Studie, und unter Vermeidung von Hypoglykämien versuchen, sollte ihnen nach aktuellem Wissensstand damit am besten gedient sein. die Mortalität erhöht Nachdem die Studie von Ridc in der Öffentlichkeit häufig nicht diskutiert wurde, zeigt die Nachanalyse der ADVANCE-Studie nun ebenfalls, dass ein niedriger $\mathrm{HbA}_{1 c}$ günstiger ist als ein zu hoher. Das neue hierbei ist jedoch, dass Schwellenwerte definiert werden konnten, unterhalb derer es nicht zu 